\title{
Whole genome copy number analyses reveal a highly aberrant genome in TP53 mutant lung adenocarcinoma tumors
}

\author{
Maria Moksnes Bjaanæs ${ }^{1,2 *}$, Gro Nilsen ${ }^{3,4}$, Ann Rita Halvorsen', Hege G. Russnes ${ }^{1,5}$, Steinar Solberg 6 , \\ Lars Jørgensen ${ }^{6}$, Odd Terje Brustugun ${ }^{1,7}$, Ole Christian Lingjærde ${ }^{1,3,4}$ and Åslaug Helland ${ }^{1,2}$
}

\begin{abstract}
Background: Genetic alterations are common in non-small cell lung cancer (NSCLC), and DNA mutations and translocations are targets for therapy. Copy number aberrations occur frequently in NSCLC tumors and may influence gene expression and further alter signaling pathways. In this study we aimed to characterize the genomic architecture of NSCLC tumors and to identify genomic differences between tumors stratified by histology and mutation status. Furthermore, we sought to integrate DNA copy number data with mRNA expression to find genes with expression putatively regulated by copy number aberrations and the oncogenic pathways associated with these affected genes.

Methods: Copy number data were obtained from 190 resected early-stage NSCLC tumors and gene expression data were available from 113 of the adenocarcinomas. Clinical and histopathological data were known, and EGFR-, KRAS- and TP53 mutation status was determined. Allele-specific copy number profiles were calculated using ASCAT, and regional copy number aberration were subsequently obtained and analyzed jointly with the gene expression data.

Results: The NSCLC tumors tissue displayed overall complex DNA copy number profiles with numerous recurrent aberrations. Despite histological differences, tissue samples from squamous cell carcinomas and adenocarcinomas had remarkably similar copy number patterns. The TP53-mutated lung adenocarcinomas displayed a highly aberrant genome, with significantly altered copy number profiles including gains, losses and focal complex events. The EGFR-mutant lung adenocarcinomas had specific arm-wise aberrations particularly at chromosome7p and 9q. A large number of genes displayed correlation between copy number and expression level, and the PI(3)K-mTOR pathway was highly enriched for such genes.
\end{abstract}

\footnotetext{
* Correspondence: mamok@ous-hf.no

Gro Nilsen started working for Telenor Group 01.06.2015.

'Department of Cancer Genetics, Institute for Cancer Research, Oslo

University Hospital-The Norwegian Radium Hospital, Oslo, Norway

${ }^{2}$ Department of Oncology, Oslo University Hospital, 4950 Nydalen Oslo,

Norway

Full list of author information is available at the end of the article
}

(c) The Author(s). 2021 Open Access This article is licensed under a Creative Commons Attribution 4.0 International License, which permits use, sharing, adaptation, distribution and reproduction in any medium or format, as long as you give appropriate credit to the original author(s) and the source, provide a link to the Creative Commons licence, and indicate if changes were made. The images or other third party material in this article are included in the article's Creative Commons licence, unless indicated otherwise in a credit line to the material. If material is not included in the article's Creative Commons licence and your intended use is not permitted by statutory regulation or exceeds the permitted use, you will need to obtain permission directly from the copyright holder. To view a copy of this licence, visit http://creativecommons.org/licenses/by/4.0/ The Creative Commons Public Domain Dedication waiver (http://creativecommons.org/publicdomain/zero/1.0/) applies to the data made available in this article, unless otherwise stated in a credit line to the data. 
Conclusions: The genomic architecture in NSCLC tumors is complex, and particularly TP53-mutated lung adenocarcinomas displayed highly aberrant copy number profiles. We suggest to always include TP53-mutation status when studying copy number aberrations in NSCLC tumors. Copy number may further impact gene expression and alter cellular signaling pathways.

Keywords: Copy number, Lung cancer, NSCLC, p53, mTOR

\section{Introduction}

Lung cancer is the most frequent cancer among men and the third most frequent cancer type among women worldwide [1]. Patients diagnosed with lung cancer have a high mortality, and the disease causes almost as many lost life-years as colon, prostate and breast cancer combined [2]. Non-small cell lung cancer (NSCLC) accounts for about $85 \%$ of all lung cancer cases, and tumors with adenocarcinoma histology are increasing in incidence. Genetic alterations found in lung adenocarcinomas are clinically important, and EGFR mutations and translocations involving $A L K$, ROS and RET genes are currently targets for therapy $[3,4]$. TP53 mutations are seen in approximately $50 \%$ of NSCLC [5], and the potential predictive and prognostic value of TP53 mutation status is debated [6]. Chromosomal abnormalities are frequent events in NSCLC tumors, and both mutations and copy number aberrations can be main drivers of the disease [7]. Specific patterns of copy number gains and losses have been associated with different cancer types [8-10], and linked to histological subtypes of lung cancer tumors [11]. In breast cancer tumors, focal complex events characterized by multiple closely spaced aberrations seen in genome-wide copy number profiles, have been described and associated with prognosis [12]. Focal complex events has been reported to be more frequent in lung adenocarcinomas compared with other histological subtypes of lung cancer tumors [11], but thorough studies of chromosomal architecture in NSCLC tumors are lacking. Different copy number profiles in lung adenocarcinoma tumors with and without mutations in EGFR or KRAS have been described [13-15], but information about structural events have not been included in these studies. The effect of copy number aberrations on carcinogenesis is complex and some reports have shown that the expression of genes located in chromosomal regions involved in copy number alterations varies consistently with the DNA copy number [16] suggesting that these alterations can affect the expression of oncogenes and/or tumor suppressor genes $[8,9]$. The affected genes may further act together to alter cellular signaling pathways in the malignant cells $[17,18]$.

In this study, we aimed to characterize the genomic architecture of the NSCLC tumors. Copy number data were obtained by high-resolution SNP arrays on 190 tumor samples from operable NSCLC patients. We further analyzed the complexity of the tumor genomes based on the allele-specific copy number profiles. Substantial differences were found between subgroups of samples when stratified on the basis on histology, smoking history, EGFR-, KRAS- and particularly TP53 mutation status. Furthermore, by integrating gene expression data from a subset of 113 lung adenocarcinoma samples, we identified genes for which the expression was affected by copy number and subsequently identified the cellular pathways most enriched for such genes.

\section{Material and methods}

\section{Ethic statement and patients included in the study}

This project was approved by the institutional review board and regional ethics committee (S-05307). Participants included were patients with operable lung cancer admitted to the cardio-thoracic surgery department at Oslo university hospital-Rikshospitalet, from 2006 to 2011. All patients received oral and written information about the project and signed a written consent before entering the study. Clinical data were obtained from questionnaires, medical records, histology reports and the Cancer registry of Norway.

The tumor tissue was snap frozen in liquid nitrogen and stored at $-80^{\circ} \mathrm{C}$ until DNA and RNA isolation. Genomic DNA was extracted from the frozen tumor tissue using the Maxwell ${ }^{\circ} 16$ DNA purification kit following standard protocol and RNA was extracted with standard TRIZOL methods (Invitrogen, Carlsbad, CA, USA) as previously described $[19,20]$. EGFR mutation analyses of exons 18-21 were performed by using the TheraScreen EGFR mutation kit and the KRAS mutations were tested by using the wobble-enhanced ARMS (WE-ARMS) method [21]. TP53 mutations in exon 2-11 were analyzed by Sanger sequencing using the AB 3730 DNA Analyzer (Applied Biosystems) after standard protocol as previously described [20].

\section{SNP arrays and mRNA expression arrays}

DNA was hybridized to Affymetrix Genome-Wide $\mathrm{Hu}$ man SNP 6.0 arrays following the manufacturer's instructions (Affymetrix, Santa Clara, CA) at AROS Applied Biotechnology (Aarhus, Denmark). A subset of the lung adenocarcinoma samples $(n=113)$ had mRNA expression data available. This was assessed using gene expression microarrays from Agilent technologies 
(SurePrint G3 human GE, $8 \times 60 \mathrm{~K}$ ) as previously described [20]. The mRNA data were log2-transformed and normalized between arrays by using the 75th percentile method in Genespring GX analysis Software v.12.1 (Agilent technology). The mRNA expression array includes 42,066 unique probes, and 30,370 probes remained after filtering out probes with no gene annotation or available gene names. The average gene expression value was calculated when a gene was mapped with more than one probe at the array. 22,076 unique genes remained for further analyses.

\section{Statistical analyses \\ Copy number segmentation and estimation of allele-specific copy numbers}

The SNP data (Affymetrix CEL-files) were pre-processed by using the Affymetrix Power Tools (APT) software and the PennCNV software [22] to obtain total signal intensities $(\log \mathrm{R})$ and $\mathrm{B}$ allele frequencies (BAF) at each genomic marker. All samples were normalized to a custom-made cohort of normal samples from the HapMap project, the 1000 Genomes Project and the Wellcome Trust Case-Control Consortium [23-25]. After adjusting $\log \mathrm{R}$ for $\mathrm{GC}$ binding artifacts [26], the $\log \mathrm{R}$ and BAF results were used as input for the allele-specific segmentation of normalized raw data with ASPCF [27] with penalty parameter $\gamma=50$ and the subsequent analysis with the ASCAT (Allele-Specific Copy number Analysis of Tumors) algorithm (version 2.3) [28]. The result was an allele-specific copy number profile of each tumor as well as estimates of tumor ploidy and tumor cell fraction (cellularity). In five samples, ASCAT failed to come up with a solution, and these samples were excluded from further analyses.

\section{Identification of recurrently aberrant regions}

From ASCAT we obtain for each sample a segmentation of the tumor genome, where each segment has a start and end position in the genome and two allele-specific copy number values $(0,1,2, \ldots .$.$) corresponding to the$ two alleles. The sum of the two copy number values represents the total copy number of the segment, and the median total copy number across the whole genome (taking into account the size of each genomic segment) represents a measure of tumor ploidy. Segments with total copy number exceeding the ploidy were called as gains, while segments with total copy number less than the ploidy were called as losses. The identified aberrations among all NSCLC samples were plotted with the frequency of samples with segments called as gain or loss (in the $y$-axis) at every genomic position ( $x$-axis). Segments that were aberrant in a fixed proportion $\mathrm{p}$ of the samples were defined as recurrent aberrant regions and were matched by position to an annotation file with information of gene names, cytobands and chromosomal regions (in this paper, we used $p=0.30$ ).

\section{Quantification of genomic complexity from copy numbers}

To more thoroughly examine the segmental and structural DNA aberrations, we applied a bioinformatic method that compresses the allele-specific copy number profile into a small number of score values capturing the degree of presence of features in the profile. The algorithm compresses the allele-specific copy number profile in a specified genomic region $\mathrm{R}$ into eight scores that reflect different aspects of the genomic complexity. In this paper, the genomic region $\mathrm{R}$ was either the whole genome (resulting in eight scores per tumor) or a chromosome arm (resulting in $8 \times 43=344$ scores per tumor when applied to all 43 arms separately). The scores reflect level of variation relative to the median copy number in the region (var), level of steep transition in copy number (steep), level of curvature or oscillation in copy number (curv), level of deviation from the genome ploidy (dev), level of gain relative to ploidy (gain), level of loss relative to ploidy (loss), level of allelic skewness attributable to loss of heterozygosity (loh), and level of non-LOH related allelic skewness (asym). Figure 1 gives a visual guide to the type of features this algorithm scores can capture in a copy number profile. Note that the first six scores are calculated based on total copy numbers, while the last two use the allele-specific copy numbers. All scores take the magnitude as well as the width of aberrant regions into account. The first score, var, will be large if there are local variations in copy

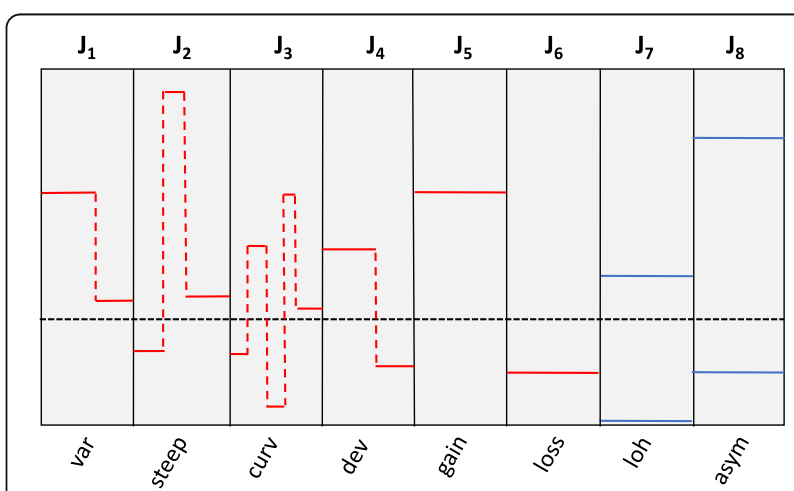

Fig. 1 An overview of the eight indices in the algorithm. Var: variation relative to median copy number (J1), steep: steep transitions (J2), curv: curvature or oscillation (J3), dev: deviation from ploidy (J4), gain: gain relative to ploidy (J5), loss: loss relative to ploidy (J6), loh: $\mathrm{LOH}$ related allelic skewness (J7), and asym: non-LOH related allelic skewness (J8). The scores can be detected genomewide or arm-wise in each sample. The red curves represent total copy numbers, while the blue curves represent the number of copies of each allele. Ploidy is defined as the median genome copy number and is shown as a dashed horizontal line 
number. The score steep reflect if there are narrow copy number shifts of some magnitude, while the score curv reflects such events that are also oscillating. Both steep and curv are associated with focal complex events. The fourth score, dev, detects the amount of deviation from the genome ploidy, while the scores gain and loss reflect whether such deviations correspond to copy number gain or loss, respectively. Note that the scores gain and loss are different from the gains and losses defined in the previous paragraph as both the magnitude and the width of the aberrant regions go into the calculation of the former whereas the latter simply reflect an indicator of whether a segment is aberrant or not. Both scores loh and asym are triggered by regions where there is skewness in the number of copies of the two alleles. The former is, however, restricted to only reflect events where one of the alleles is completely lost, while the latter detects events where this is not the case. The genome-wide and arm-wise scores were further applied to analyze the lung cancer samples stratified into defined subgroups. The bioinformatic method used in this paper is a variant of the CARMA algorithm [29].

\section{Statistical hypothesis tests}

Most statistical analyses were performed by using the $\mathrm{R}$ computing framework [30] and SPSS (v.21). T-tests were applied to perform two-sample comparisons of means, and the non-parametric Wilcoxon rank-sum test was used when the T-test was not appropriate because of strong deviations from normality of the data. $P$-values < 0.05 were considered statistically significant and Bonferroni corrections for multiple testing were performed when appropriate.

\section{Integration of gene expression and copy number data; cis- associated genes}

To focus on genes for which copy number is likely to affect gene expression, we integrated copy number data with mRNA expression data obtained in a subset of 113 lung adenocarcinoma samples. To obtain matched copy number and gene expression values for each sample, we found for each gene expression probe its location in the genome and the overlapping copy number segment as found by ASCAT. The value of the expression probe and the estimated total copy number of the segment then formed a matched pair, and this calculation was performed for all gene expression probes.

To identify genes likely to be substantially influenced by copy number, a combination of two criteria were used and the resulting genes were referred to as cisgenes. First, the Pearson correlation between the copy number value and the gene expression should exceed 0.4 . Second, at least one of the following two t-tests should be significant $(P<0.05$, with no adjustment for multiple comparisons). The first test compares the expression level of samples with loss and samples with normal copy number, while the second test compares the expression level of samples with normal copy number and samples with gain (where loss and gain are defined as described previously). For the purpose of visualization, the smaller of the two $P$-values was rescaled to $\mathrm{Z}$-scores by applying the transformation $\mathrm{Z}=$ $-\mathrm{F}^{-1}$ (Pval), where $\mathrm{F}$ is the cumulative standard normal distribution. P-values are uniformly distributed between 0 and 1 and the Z-score will follow a standard normal distribution; furthermore, $P<0.05$ corresponds to $\mathrm{Z}>$ $\mathrm{F}^{-1}(0.05)=1.64$. Note that the tests above were designed to be liberal in order to also capture genes that were moderately associated with copy number; hence there was no correction for multiple comparisons above.

\section{Pathway analysis}

Ingenuity Systems Pathway Analysis (IPA) was applied to the list of cis-genes to derive the most enriched pathways associated with the genes regulated by copy number. A core-analysis was assessed to find the level of representation of our selected genes in already defined canonical pathways. The significance of the association between the cis-gene list and the pathways were tested with Fisher's exact tests, and the Benjamini-Hochberg correction for multiple testing was applied.

\section{Results}

High-resolution SNP arrays were used to obtain copy number data for 200 NSCLC samples. Five samples were excluded after pathological re-examination and five samples failed to come up with a solution in the ASCAT algorithm, leaving 190 NSCLC samples for further analyses.

The majority of samples were adenocarcinomas $(n=$ 154) while squamous cell $(n=32)$ and large cell $(n=4)$ histology were also represented. To avoid picking up histological differences, only the adenocarcinoma samples were included in the subgroup and integration analyses. All the 20 EGFR-mutated tumors were of adenocarcinoma histology and the patients with EGFRmutated tumors were mainly women $(16 / 20)$ and neversmokers $(12 / 20)$. The patients with KRAS-mutated tumors were both men (21/55) and women (34/55), and most of them were current- or former smokers (52/55). TP53 mutations were also present in both men and women $(43 / 79,36 / 79)$, and $11.4 \%$ were never-smokers. The EGFR and KRAS mutations were mutually exclusive. 12 of the patients had double mutations including KRAS and TP53 and 10 patients had both EGFR- and TP53mutations. The main clinical and molecular characteristics are shown in supplementary Table 1. 


\section{Recurrent aberrations, tumor ploidy and aberrant cell fraction}

The ASCAT algorithm estimates the fraction of aberrant cells and allows the determination of tumor ploidy for each sample. The median tumor ploidy across all samples was 2.71 (range 1.51-5.49), with the highest proportion of samples with a ploidy close to $2 \mathrm{~N}$, a second prominent peak at 3.5 and a third peak at 5.5 (supplementary Fig. 1). The mean estimated aberrant cell content was $53.3 \%(21-100 \%)$. There were no statistically significant differences in tumor ploidy or aberrant cell fraction between histological subtypes, EGFR-mutated/ wild type, KRAS-mutated/wild type or stratified by stage or smoking status. The tumors with TP53 mutations had a higher tumor ploidy $(p=0.012)$ and a smaller fraction of aberrant cells $(p=0.007)$ compared with the TP53 wild type tumors.

The NSCLC tumor tissue displayed overall complex DNA copy number profiles with numerous recurrent alterations observed in almost all chromosomes (Fig. 2). Gains were enriched in chromosome arm 1q, 3q, 5p, 6p, $7 \mathrm{p} / \mathrm{q}, 8 \mathrm{q}, 14 \mathrm{q}, 17 \mathrm{q}, 19 \mathrm{q}$ and $20 \mathrm{p} / \mathrm{q}$, with chromosome arm $1 \mathrm{q}$ and $5 \mathrm{p}$ as the most frequently gained regions. Losses were located at $1 \mathrm{p}, 3 \mathrm{p}, 4 \mathrm{p} / \mathrm{q}, 5 \mathrm{q}, 6 \mathrm{q}, 8 \mathrm{p}, 9 \mathrm{p} / \mathrm{q}$, $10 \mathrm{q}, 11 \mathrm{p}, 13 \mathrm{q}, 14 \mathrm{q}, 15 \mathrm{q}, 16 \mathrm{q}, 17 \mathrm{p}, 18 \mathrm{q}, 19 \mathrm{p}, 21 \mathrm{q}, 22 \mathrm{q}, \mathrm{X}$ and $Y$, with $3 p$ and $X$ as the regions with the highest frequencies of loss. Several of the alterations were found across histological subtypes. The recurrently aberrant regions included genes implicated in the pathogenesis of NSCLC with known oncogenes such as MDM4 (1q), RIT1 (1q), DROSHA (5p), PIK3CA (3q), EGFR (7p), and $N K X 2-1(14 \mathrm{q})$, and tumor suppressor genes such as TP53 (17p). A more detailed overview of recurrent chromosomal aberrations ( $>30 \%$ of the tumors)

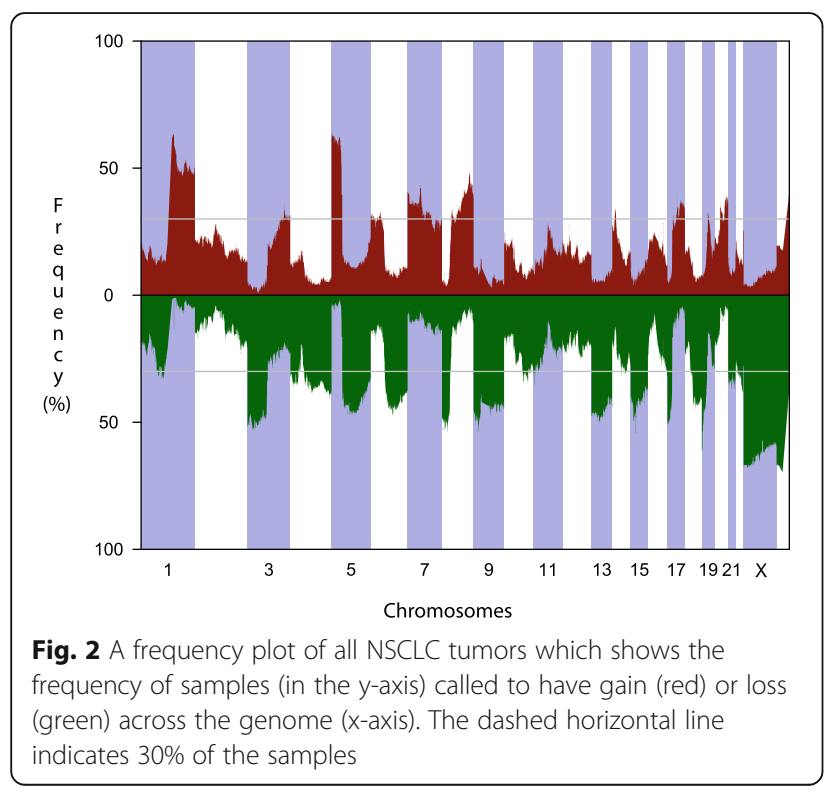

including positions, cytobands and genes located in these regions are found in supplementary Table 2, and an overview of the aberrations is listed up in supplementary Table 3.

\section{DNA copy number profiles between subgroups of samples}

To study the differences in DNA allele-specific copy number profiles between NSCLC tumors based on histology and lung adenocarcinoma samples stratified by smoking history, EGFR-, KRAS- and TP53 mutation status, we used genome-wide and arm-wise scores. As explained in the methods section, this algorithm compresses the allele-specific copy number profile into eight scores which captures complementary facets of the copy number profiles. Examples of arm-wise scores calculated for two selected copy number profiles are depictured in supplementary Fig. 2.

By using the genome-wide scores for all eight indices, no differences were identified between the adenocarcinomas and squamous cell carcinomas (supplementary Fig. 3a). At the arm-wise level, the histological subtypes differed at chromosome arm 1q, 3q, 5q, 6q, 12p, and 19q. The adenocarcinomas had a significantly higher gain-score at chromosome arm $1 \mathrm{q}$ and $5 \mathrm{q}$, and a greater loss- and $\mathrm{LOH}$ score at $6 \mathrm{q}$ and $12 \mathrm{p}$. The chromosome $\operatorname{arm} 3 q$ had significantly higher gain- and asymmetryscores in the squamous cell carcinomas. Focal complex events were observed in the adenocarcinomas at $19 q$ (curv) and were combined with LOH. The dev score detects the amount of deviation from the genome ploidy and did also pick up the difference in copy number at chromosome arm1q and 3q (supplementary Fig. 3b and 3c).

The most striking findings in the subgroup-analyses were the large difference at all genome-wide indices between the TP53-mutated compared with the TP53 wild type lung adenocarcinomas (Fig. 3a). All eight genomewide scores were significantly higher in the TP53-mutated lung adenocarcinomas. In the arm-wise analysis, we identified significantly higher scores in at least one index in nearly all chromosome arms (detailed information in supplementary Table 4). The chromosome arm $17 \mathrm{p}$, which includes the location of TP53, had significantly more loss and $\mathrm{LOH}$ in the TP53-mutated lung adenocarcinomas (Fig. 3b). When we studied the exact location of the TP53 gene at 17p, we found that 55 of the 58 lung adenocarcinomas with TP53 mutation had loss of the wild type TP53 locus (94.8\%). The remaining mutated allele was frequently amplified in the TP53-mutant tumors. Of the TP53 wild type tumors, 27 of 96 (28.1\%) had TP53 LOH, which is a significantly lower frequency than the TP53-mutated samples. No samples had homozygote deletion of the TP53 gene. 

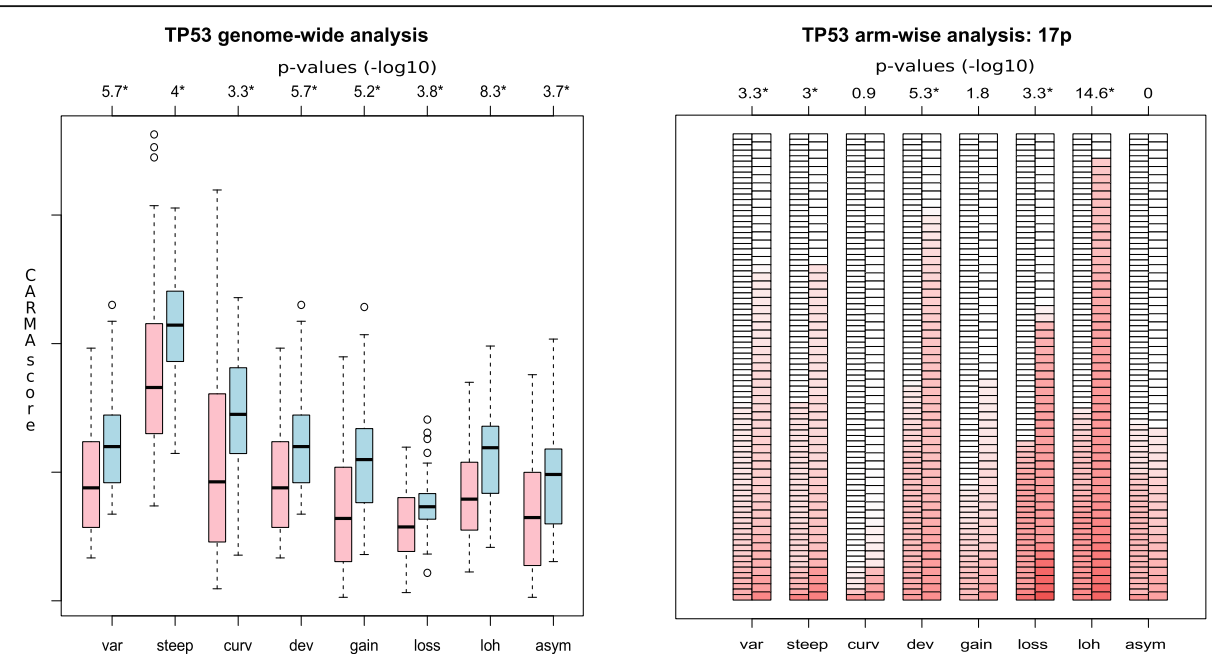

Fig. 3 a All eight scores were significantly higher in the TP53 mutated tumors compared with the TP53 wild type tumors in the genome-wide analysis. The box plot shows the genome-wide scores for the TP53-mutated tumors (blue) and the TP53 wild type tumors (pink) for all eight indices. The $p$-values $(-\log 10)$ from the comparison analyses are shown above each column and a value $>2$ was considered as significant after multiple testing corrections. $\mathbf{b}$ The arm-wise scores at chromosome arm 17p showing the eight indices for the TP53 wild type tumors at the left and the TP53 mutated tumors at the right. The scores for each sample are colored by intensity. The p-values (-log10) from the comparison analyses are shown above each column and a value $>3$ was considered as significant after multiple testing corrections

When we stratified the samples based on differences in EGFR mutation status, we did not identify differences at the genome-wide level (supplementary Fig. 4a). By investigating arm-wise differences, we found that the EGFR wild type tumors had a significantly higher loss-score at chromosome arm $7 \mathrm{p}$. The gain-score at $7 \mathrm{p}$ was borderline significantly higher in the EGFR mutated tumors but was also frequently amplified in the EGFR wild type tumors (supplementary Fig. 4b). The EGFR wild type tumors had more gain at $9 \mathrm{q}$ and $\mathrm{LOH}$ in $4 \mathrm{p}$ and $11 \mathrm{q}$, and significantly more focal complex events at 3p, 5p, 11q and 12q captured by the curv-score. Zooming in at the genomic location of the EGFR gene, we found that both the EGFRmutated samples and the EGFR wild type adenocarcinomas had an increased number of copy numbers at this position, but that the EGFR-mutant tumors had a significantly higher number of copies compared with the EGFR wild type tumors $(p=0.001)$ (Fig. 4).

Stratifying the samples by KRAS mutation showed significantly higher genome-wide scores on steep and curv in the KRAS wild type tumors, reflecting generally more focal complex events in these tumors (supplementary Fig. 5). This was also seen in the arm-wise analysis with more focal complex events in the KRAS wild type tumors at chromosome arm $2 \mathrm{p}$, $11 \mathrm{p}, 11 \mathrm{q}$, and $18 \mathrm{q}$. Comparing tumors from neversmoking patients and former smokers/smokers did not identify significant differences at the genome-wide level. At the arm-wise level tumors from former smokers/smokers had significantly more focal complex events at chromosome $2 \mathrm{q}$ and $3 \mathrm{q}$. Tumors from former smoker/smokers had additionally higher lossscores at $5 \mathrm{q}$ and $7 \mathrm{p}$ and higher gain-score at $12 \mathrm{p}$.

The differences between subgroups of samples are summed up in supplementary Table 4.

\section{Cis-associated genes and affected pathways}

The integrative analysis was assessed to identify genes with expression influenced by the copy number. Among

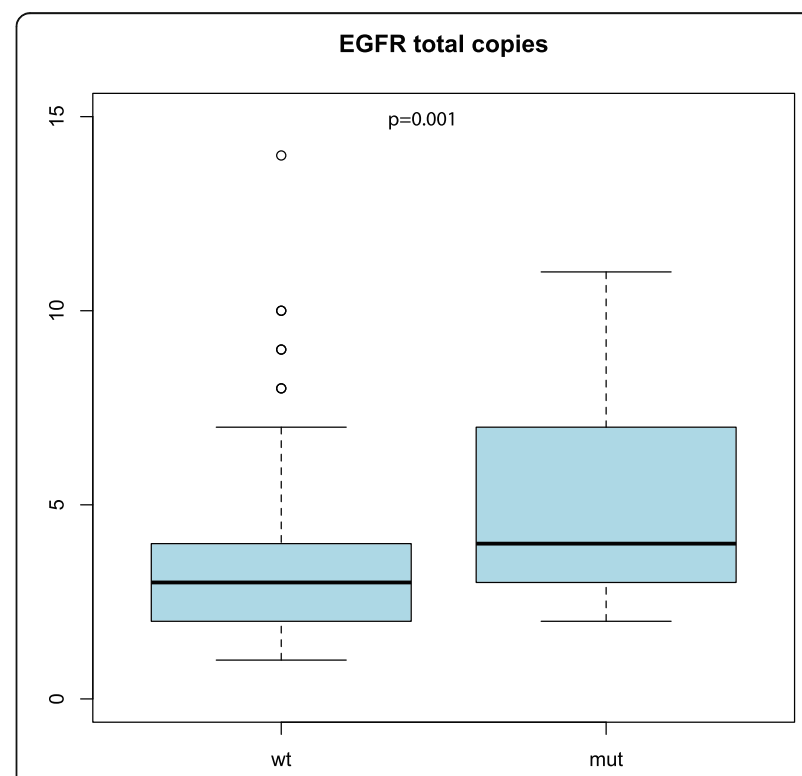

Fig. 4 The box plot shows the total copy number at the location of the EGFR gene. The EGFR-mutant samples had significantly more copies than the EGFR wild type tumors $(p=0.001)$ 
the 22,076 genes profiled, a total of 2868 genes abided the two criteria and were identified as cis-genes (both correlated in cis with a coefficient $>0.4$ and a significant difference in gene expression between samples with copy number gain versus normal or loss versus normal) (supplementary Fig. 6). These genes were localized throughout the genome, but particularly in the recurrently aberrant regions which includes 1668 of the cis-genes identified. Genes such as EGFR, PIK3CA, DROSHA, $M D M 4$, and $A P C$ were located inside recurrently aberrant regions, while other known cancer-genes such as NF1, MET and $m T O R$ were identified as cis-genes and located outside the most recurrently altered regions. Using Ingenuity Pathway Analysis, signaling pathways associated with the copy number driven cis-genes were identified (supplementary Table 5). Interestingly, a large part of the cis-genes was associated with gene expression, post-transcriptional modifications and post- translational modifications, identified as the top molecular and cellular functions from the IPA-analysis. Among the top significantly associated pathways were the EIF2( $p$-value $<1.02 \cdot 10^{-8}$ ) and the mTOR-signaling pathway (p-value $<8.82 \cdot 10^{-4}$ ), which included 57 and 45 cisgenes respectively. PIK3CA is a key molecule in both pathways, and this gene was identified as a cis-gene with significantly higher expression in tumors with gain compared with normal copy number at the position of the PIK3CA gene. In the mTOR-pathway a large number of important genes were among the identified cis-genes such as mTOR, AKT, KRAS, RPS6, RAPTOR, EIF4G1 and RPS6KB1 (p70S6K) (Fig. 5). In the EIF2-pathway most of the EIF-genes (Eukaryotic translation initiation factor) were differentially expressed between the gain/ normal and the ribosomal protein genes were differentially expressed between the loss/normal. $68 \%$ of the cisgenes in the EIF2 pathway and $64 \%$ of the cis-genes in

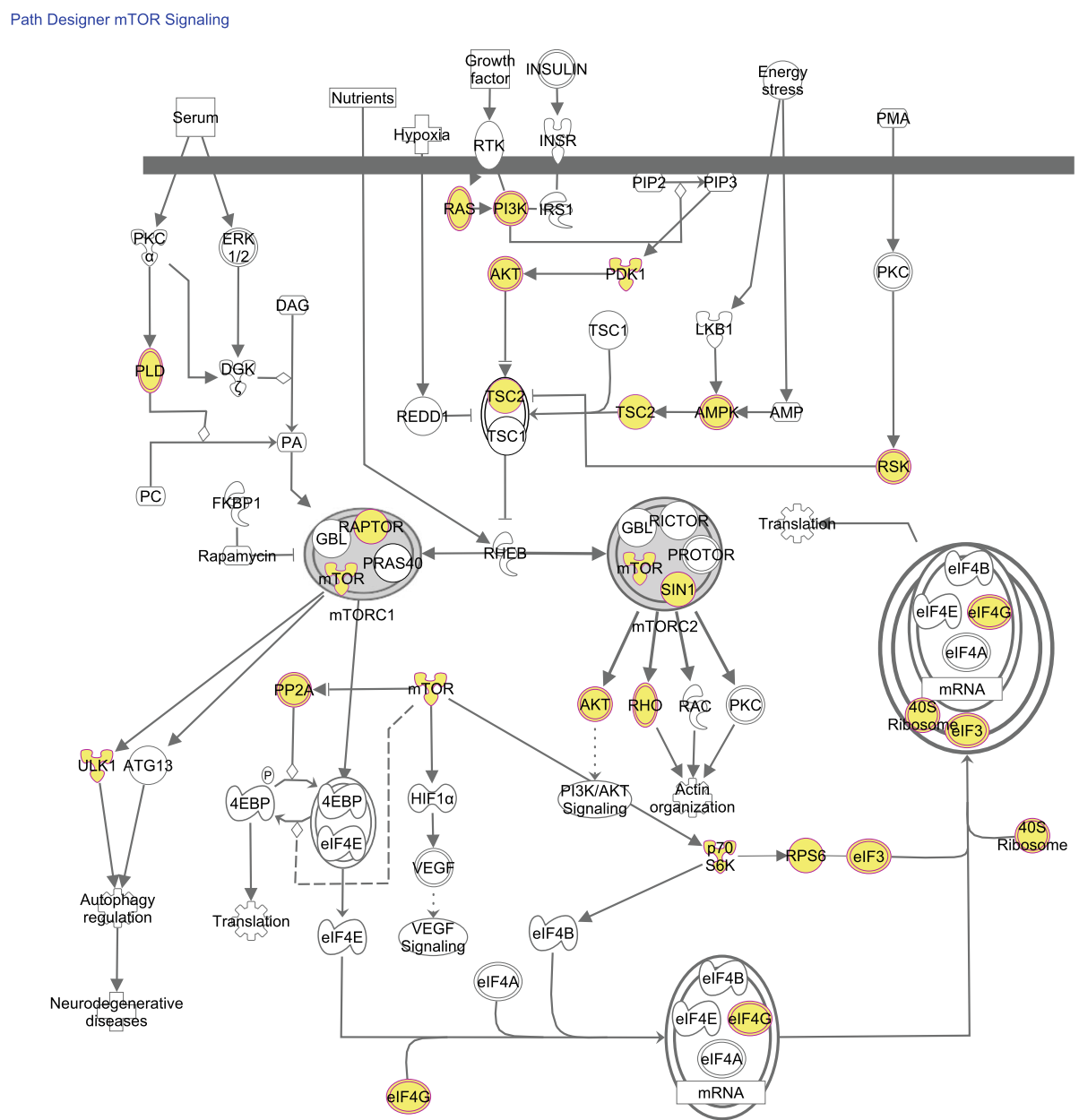

(1) 2000-2015 QIAGEN. All rights reserved.

Fig. 5 Ingenuity Pathway Analysis was used to identify cellular signaling pathways related to the identified cis-genes (genes with expression influenced by the copy number) in lung adenocarcinoma tumors. The mTOR pathway was one of the top signaling pathways in this analysis and the molecules colored yellow were among the genes identified as cis-genes 
the mTOR pathways were located in recurrently aberrant regions.

\section{Discussion}

The current study presents an exploratory wholegenome investigation of copy number alterations including the genomic architecture of NSCLC tumors. The copy number data were obtained using high-resolution SNP arrays of 190 early-stage NSCLC tumors. By stratifying the samples into biological relevant subgroups, we identified large differences particularly in the TP53mutated tumors that displayed a considerable number of gains, losses and focal complex events both in the genome-wide and arm-wise analyses. Integration of DNA copy number with mRNA expression data showed that genes with expression influenced by the copy number were associated with important cellular signaling pathways previously not known to be driven by copy number change.

Overall, the NSCLC tumor tissue displayed complex DNA copy number profiles with many gains, losses and focal complex events throughout the genome. The global copy number profile was comparable to those seen in similar studies of NSCLC tumors [10,31,32]. The most common regions with gains were located at chromosome arm 1q and 5p. Chromosome arm 1q includes MDM4 and RIT1 genes, and by integrating mRNA data, we found that expression of MDM4 correlated with increased copy number leading to up-regulation of the expression of this gene. MDM4 is important in carcinogenesis, and increased MDM4 activity can suppress the TP53 activity allowing the cancer cells to proliferate [33]. Mutation in RIT1 has recently been identified in lung adenocarcinomas and RIT1 is proposed to be a driver oncogene in a specific subset of lung adenocarcinomas [34]. This gene was recurrently gained in our data and may be an alternative path for oncogenic activation. The commonly gained $5 \mathrm{p}$ region includes DROSHA whose mRNA expression is correlated in-cis in our data. This gene is a crucial regulator of microRNA expression, and increased expression of DROSHA has been linked to poor prognosis in lung cancer $[35,36]$. Other recurrently gained regions were $7 \mathrm{p}$ that includes the EGFR gene, and this region was frequently gained both in EGFR-mutated and wild type tumors. Chromosome arm $3 p$ was lost in a high proportion of samples. This is a known event in lung cancer tumors, and was among the first chromosomal abnormality to be recognized originally identified by karyotyping [37].

Despite distinct histological differences, squamous cell carcinomas and adenocarcinomas show remarkable similar copy number patterns with no significant differences in genome-wide scores. In the arm-wise analysis, however, squamous cell carcinomas had a higher gain score at $3 \mathrm{q}$ as compared with adenocarcinomas. The squamous cell carcinomas also have significantly increased asymmetry-score at 3q, indicating an asymmetric gain in this chromosome arm. The lung adenocarcinomas had significantly more gain at chromosome arm $1 \mathrm{q}$ and $5 \mathrm{q}$ and a greater loss combined with $\mathrm{LOH}$ at $6 \mathrm{q}$ and $12 \mathrm{p}$. The copy number differences between squamous cell carcinomas and the adenocarcinomas of the lung have been studied by others, and the increased gain at $3 \mathrm{q}$ in the squamous cell carcinomas have been reported in several studies [38, 39]. The increased gain at $5 \mathrm{q}$ in the lung adenocarcinomas has also been reported previously by Staaf et al. [11]. This study is the only other study which has included focal complex events in NSCLC tumors, and they identified more focal complex events in squamous cell carcinomas. This was not confirmed in our data, but the limited number of squamous cell carcinomas included in this study might serve as an explanation. Furthermore, we find that gain often occur together with asymmetry, indicating that gain often is an asymmetric event with respect to the two alleles in NSCLC tumors. Similarly, loss and LOH often co-occur when the loss is not a copy neutral event.

Mutations in the TP53 gene are common events in lung cancer tumors. The TP53-mutated tumors had significantly higher ploidy, estimated by the ASCAT algorithm, compared with the TP53 wild type tumors. After adjusting for estimated ploidy and aberrant cell count, we performed comparison analyses and found that the TP53-mutated lung adenocarcinomas had a significantly higher score at all eight indices in the genome-wide analysis (Fig. 3). The lung adenocarcinomas with mutant TP53 gene have generally more segments deviating from the median ploidy with consistently more gains and losses throughout the genome. The TP53-mutant tumors additionally have more focal complex events captured by the steep and curv scores, which may be contributing to the aggressive phenotype associated with the TP53 mutations seen in other cancer types [40]. The genome-wide $\mathrm{LOH}$ and asymmetry scores were additionally significantly higher in the TP53-mutated tumors. Particularly interesting is it that nearly all TP53mutated tumors had TP53 LOH, indicating that inactivation of both TP53 alleles are important as proposed in Knudsons two-hit hypothesis [41]. The $\mathrm{LOH}$ events in TP53-mutated tumors were often accompanied by copy number gain of the mutant allele. In the TP53 wild type tumors, the TP53 LOH was seen in $31.4 \%$ of the samples, suggesting a dysfunction in the TP53-pathway in a large amount of all lung adenocarcinoma tumors.

The high genome-wide scores in the TP53 mutated tumors indicate a highly unstable genome. Other studies have demonstrated how TP53 mutation status might 
reflect tumor mutation burden, and association with longer overall survival in patients receiving immunotherapy [42]. This reflects that the well-known TP53 mutation status might be clinically important also in the future. The finding of the complex copy number profiles in TP53-mutant lung adenocarcinomas in our study is very convincing, and we suggest that TP53-mutation status should be considered implemented for biological stratification purposes, in studies involving genomic aberrations.

Lung adenocarcinomas with EGFR mutation comprise a specific clinical subtype and are more frequent in women, never-smokers and patients with Asian ethnicity [43]. To better understand the biology of the EGFR-mutated lung adenocarcinomas we compared copy number profiles between EGFR-mutated and wild type tumors. In the chromosome arm-wise analysis, we identified alterations of chromosome arm $7 p$ that were gained in both EGFR-mutated and EGFR wild type tumors, and significantly more lost in the EGFR wild type tumors. The EGFR gene is located at $7 \mathrm{p} 12$, and this region was also significantly gained in both EGFR wild type and $E G F R$-mutated tumors, but with a significant higher number of total copies in EGFR-mutated tumors (Fig. 4). When integrating mRNA expression data, we found that the EGFR mRNA expression was correlated with gained copy number, and previous studies have also shown that the copy number alterations in chromosome 7 are correlated with protein expression and activation of the EGFR pathway [44]. EGFR mutation is a strong predictive biomarker for tyrosine kinase inhibitor response. It is however debated if copy number gain may act as a predictive marker for EGFR-TKI response in patients with EGFR wild type lung cancer tumors $[45,46]$. The aberrations of chromosome arm $7 \mathrm{p}$ seem to be consistent with previous reports [13, 14, 47]. We also found that the EGFR wild type lung adenocarcinomas had significantly more gain at 9q, an aberration difference not described earlier. Other copy number differences in gains and losses between EGFR-mutated and wild type tumors have been described previously [13, 14, 47], but were not validated in our study. The lack of consistency may be caused by small sample sizes and the use of different methods to call gains/losses. The scores in our analyses include both the magnitude and the width of the aberrant region into the calculation of gains and losses, which makes it challenging when comparing the results with other studies. To our knowledge, focal complex events have not been described in relation to EGFR, KRAS and TP53 mutation status, and the clinical impact of such events in lung cancer tumors is not known. The arm-wise analysis identified more focal complex events (reflected in steep and curv scores) in the EGFR wild type tumors with significantly higher curv scores at 3p, 5p, 11q and 12q. The same trend was seen in the KRAS wild type tumors, which both had significantly higher steep and curv scores at the genome-wide analysis and at specific chromosome arms. The EGFR- and KRAS wild type adenocarcinomas had additionally significantly more arm-wise aberrations compared with the EGFR- and KRAS-mutant lung adenocarcinomas, suggesting that tumors without mutational activation of these oncogenic pathways are more driven by copy number aberrations than of point mutations. The TP53-mutated tumors had the opposite pattern with more focal complex events in the tumors harboring a TP53 mutation. This was consistent with the findings in the pan-cancer study by Ciriello et al., which found TP53 mutations enriched in the C-class (copy number driven) tumors [7].

The aberrational pattern is similar across different studies of NSCLC tumors. Previous studies have shown that the expression of genes located in chromosomal regions involved in gains or losses varies consistently with the DNA copy number $[48,49]$. We approached this by first investigating how the gene expression is affected by copy number alterations and secondly to study whether any known cellular pathways are overrepresented in the list of affected genes and hence probably regulated by copy number. Two of the most significantly affected pathways were the $m T O R$ - and EIF2- signaling pathways, both related to the PIK3CA gene. Mutations of the PIK3CA gene occur in lung adenocarcinoma tissue, but is a relatively seldom event [50]. We found PIK3CA frequently gained and the gene expression significantly correlated with gain in copy number. Among the genes associated with the $m T O R$-pathway, forty-five were cisgenes. The expression of several important oncogenes such as $A K T 1, A K T 2$, and KRAS were positively correlated with the copy number in our analysis. The $m T O R$ gene and several of its effectors (RPS6KB1 ( $p 7056 K)$, RPS6, and EIF4G1) were also altered. The PI(3)K-mTOR pathway was one of the key pathways found activated at a protein level in a large lung adenocarcinoma study by TCGA [51]. In this paper the activation of the pathway was partly explained by mutations (in PIK3CA or STK11), but some samples with increased pathwayactivation lacked known underlying mechanisms. We suggest that altered expression of cis-genes affected by underlying copy number aberrations may increase the activity of this pathwayDrugs that target the mTOR pathway have shown interesting results in other cancer types [52], which highlight its clinical importance. Clinical trials in lung cancer targeting the PI(3)K-mTOR pathway have shown variable responses when given as monotherapy [53-55]. The lack of responses in some patients may be due to the complex regulation of the pathway and interplay with other oncogenic pathways [54]. 
The chromosomal structure in lung carcinomas is highly aberrant and copy number alterations in tumor or in cell-free DNA might predict response to immunotherapy in cancer patients [56]. The findings in this study encourage further research of whole genome copy number alterations and to increase the biological understanding and of therapeutic approaches targeting the $\mathrm{PI}(3) \mathrm{K}$ mTOR pathway.

\section{Conclusion}

Knowledge of molecular alterations in cancer is rapidly increasing, and it can be challenging to get an overview of the information and interpret the biological relevance of the data. In this study we studied common copy number events including the genomic architecture in NSCLC tumors and differences between subgroups of samples. The TP53-mutated lung adenocarcinomas showed highly aberrant copy number profiles in both genomewide and chromosome-arm analyses and TP53 mutation status should always be considered included in studies of copy number aberrations in tumors tissue. Furthermore, nearly all TP53-mutated tumors had lost the second TP53 allele, and this was also a frequent event in the TP53 wild type tumors. The arm-wide analysis with samples stratified by the EGFR mutation status, revealed differences particularly at chromosome arm $9 \mathrm{q}$ and $7 \mathrm{p}$ involving the EGFR gene. To better understand the potentially functional effects of copy number aberrations we performed integrative analyses including copy number and mRNA expression data. By this approach we identified cis-genes whose expression correlated with copy number and further were associated with important oncogenic pathways such as the $\mathrm{PI}(3) \mathrm{K}-\mathrm{mTOR}$ pathway.

\section{Supplementary Information}

The online version contains supplementary material available at https://doi. org/10.1186/s12885-021-08811-7

\section{Additional file 1.}

Additional file 2. Recurrent aberrant regions and associated genes.

\section{Acknowledgements}

We want to thank Ingjerd Solvoll for collecting tissue samples and clinical information.

\section{Authors' contributions \\ Conception and design $(\mathrm{MB}, \mathrm{OCL}, \AA \mathrm{H})$, including patients for the study and provision of samples (SS, LJ), acquisition of data $(M B, A R H, \AA ̊ H)$, bioinformatics (GN, HGR, OCL), methods analysis and interpretation of data (GN, MB, OCL, $\AA \mathrm{H})$, drafting of the manuscript $(\mathrm{MB}, \mathrm{OCL}, \AA \mathrm{AH})$, critical revision of the manuscript (all authors) and study supervision (OTB, ÅH). The author(s) read and approved the final manuscript.}

\section{Funding}

This work was funded in part from grants from South-Eastern Norway Regional Health Authority.

\section{Availability of data and materials}

Gene expression data is available in GEO for public release, accession code: GSE66863. Other data will be made available from the corresponding author upon reasonable request.

\section{Declarations}

\section{Ethics approval and consent to participate}

All experimental protocols were approved by the institutional review board and regional ethics committee (Regionale komiteer for medisinsk og helsefaglig forskningsetikk)(S-05307). All methods were carried out in accordance with relevant guidelines and regulations. All participants received oral and written information and signed a written consent before entering the study.

\section{Consent for publication}

Not applicable.

\section{Competing interests}

No potential conflicts of interest were disclosed.

\section{Author details}

${ }^{1}$ Department of Cancer Genetics, Institute for Cancer Research, Oslo University Hospital-The Norwegian Radium Hospital, Oslo, Norway. ${ }^{2}$ Department of Oncology, Oslo University Hospital, 4950 Nydalen Oslo, Norway. ${ }^{3}$ Department of Computer Science, University of Oslo, Oslo, Norway. ${ }^{4}$ Centre for Cancer Biomedicine, Faculty of Medicine, University of Oslo, Oslo, Norway. ${ }^{5}$ Department of Pathology, Oslo University Hospital, Oslo, Norway. ${ }^{6}$ Department of Cardiothoracic Surgery, Oslo University Hospital, Oslo, Norway. ${ }^{7}$ Section of Oncology, Vestre Viken Hospital, Drammen, Norway.

Received: 3 May 2021 Accepted: 23 September 2021

Published online: 09 October 2021

References

1. Torre L, Bray F, Siegel RL, Ferlay J, Lortet-tieulent J, Jemal A. Global Cancer statistics, 2012. CA Cancer J Clin. 2015;65:87-108.

2. Brustugun OT, Møller B, Helland A. Years of life lost as a measure of cancer burden on a national level. Br J Cancer. 2014;111:1014-20. https://doi.org/1 0.1038/bjc.2014.364

3. Planchard D, Popat S, Kerr K, Novello S, Smit E., Faivre-Finn C, et al. Metastatic non-small cell lung cancer : ESMO Clinical Practice Guidelines for diagnosis, treatment Clinical Practice Guidelines. 2019;29 October 2018.

4. Herbst RS, Morgensztern D, Boshoff $C$. The biology and management of non-small cell lung cancer. Nature. 2018;553:446-54.

5. Halvorsen AR, Silwal-Pandit L, Meza-Zepeda LA, Vodak D, Vu P, Sagerup C, et al. TP53 mutation spectrum in smokers and never smoking lung cancer patients. Front Genet. 2016;7:85 may:1-10.

6. Donehower LA, Soussi T, Korkut A, Liu Y, Schultz A, Cardenas M, et al. Integrated Analysis of TP53 Gene and Pathway Alterations in The Cancer Genome Atlas. Cell Rep. 2019;28:1370-1384.e5. https://doi.org/10.1016/j. celrep.2019.07.001.

7. Ciriello G, Miller ML, Aksoy BA, Senbabaoglu Y, Schultz N, Sander C. Emerging landscape of oncogenic signatures across human cancers. Nat Genet. 2013;45:1127-33. https://doi.org/10.1038/ng.2762.

8. Zack TI, Schumacher SE, Carter SL, Cherniack AD, Saksena G, Tabak B, et al. Pan-cancer patterns of somatic copy number alteration. Nat Genet. 2013;45: 1134-40. https://doi.org/10.1038/ng.2760.

9. Beroukhim R, Mermel CH, Porter D, Wei G, Raychaudhuri S, Donovan J, et al. The landscape of somatic copy-number alteration across human cancers. Nature. 2010;463(7283):899-905.

10. Weir BA, Woo MS, Getz G, Perner S, Ding L, Beroukhim R, et al. Characterizing the cancer genome in lung adenocarcinoma. Nature. 2007; 450:893-8. https://doi.org/10.1038/nature06358.

11. Staaf J, Isaksson S, Karlsson A, Jönsson $M$, Johansson L, Jönsson $P$, et al. Landscape of somatic allelic imbalances and copy number alterations in human lung carcinoma. Int J Cancer. 2013;132:2020-31. https://doi.org/10.1 002/ijc.27879.

12. Vollan HKM, Rueda OM, Chin S, Turashvili G, Shah S, Lingjærde OC, et al. A tumor DNA complexity index is an independent predictor of survival in breast and ovarian cancer. Mol Oncol. 2014;9:1-39. 
13. Blons H, Pallier K, Le Corre D, Danel C, Tremblay-Gravel M, Houdayer C, et al. Genome wide SNP comparative analysis between EGFR and KRAS mutated NSCLC and characterization of two models of oncogenic cooperation in non-small cell lung carcinoma. BMC Med Genet. 2008;1:1-25. doi:1755-87941-25 [pii]. https://doi.org/10.1186/1755-8794-1-25.

14. Fong Y, Lin Y-S, Liou C-P, Li C-F, Tzeng C-C. Chromosomal imbalances in lung adenocarcinomas with or without mutations in the epidermal growth factor receptor gene. Respirology. 2010;15:700-5.

15. Planck M, Isaksson S, Veerla S, Staaf J. Identification of transcriptional subgroups in EGFR-mutated and EGFR/KRAS wild-type lung adenocarcinoma reveals gene signatures associated with patient outcome. Clin Cancer Res. 2013;19:5116-26. https://doi.org/10.1158/1078-0432.CCR-130928.

16. Shao X, Lv N, Liao J, Long J, Xue R, Ai N, et al. Copy number variation is highly correlated with differential gene expression: a pan-cancer study. BMC Med Genet. 2019;20:1-14.

17. Chari R, Coe BP, Vucic EA, Lockwood WW, Lam WL. An integrative multidimensional genetic and epigenetic strategy to identify aberrant genes and pathways in cancer. BMC Syst Biol. 2010;4:67.

18. Akavia UD, Litvin O, Kim J, Sanchez-Garcia F, Kotliar D, Causton HC, et al. An integrated approach to uncover drivers of cancer. Cell. 2010;143:1005-17. https://doi.org/10.1016/j.cell.2010.11.013.

19. Bjaanæs MM, Halvorsen AR, Solberg S, Jørgensen L, Dragani TA, Galvan A, et al. Unique microRNA-profiles in EGFR-mutated lung adenocarcinomas. Int J Cancer. 2014;135:1812-21. https://doi.org/10.1002/ijc.28828.

20. Bjaanæs MM, Fleischer T, Halvorsen AR, Daunay A, Busato F, Solberg S, et al. Genome-wide DNA methylation analyses in lung adenocarcinomas: association with EGFR, KRAS and TP53 mutation status, gene expression and prognosis. Mol Oncol. 2015. https://doi.org/10.1016/.j.molonc.2015.10.021.

21. Hamfjord J, Stangeland AM, Skrede ML, Tveit KM, Ikdahl T, Kure EH. Wobbleenhanced ARMS method for detection of KRAS and BRAF mutations. Diagn Mol Pathol. 2012;20:158-65. https://doi.org/10.1097/PDM.0b013e31820b4 9e2.

22. Wang K, Li M, Hadley D, Liu R, Glessner J, Grant SFA, et al. PennCNV: An integrated hidden Markov model designed for high-resolution copy number variation detection in whole-genome SNP genotyping data Genome Res. 2007;17:1665-74.

23. The Wellcome Trust Case Control Consortium*. Genome-wide association study of 14,000 cases of seven common diseases and 3,000 shared controls. Nature. 2007:447:661-78.

24. The international HapMap consortium*. The International HapMap Project. Nature. 2003:426(6968):789-96. https://doi.org/10.1038/nature02168.

25. Durbin RM, Altshuler DL, Abecasis GR, Bentley DR, Chakravarti A, Clark AG, et al. A map of human genome variation from population-scale sequencing. Nature. 2010;467:1064-7073. https://doi.org/10.1038/nature09534.

26. Diskin SJ, Li M, Hou C, Yang S, Glessner J, Hakonarson H, et al. Adjustment of genomic waves in signal intensities from whole-genome SNP genotyping platforms. Nucleic Acids Res. 2008:36:1-12.

27. Nilsen G, Liestøl K, Van Loo P, Moen Vollan HK, Eide MB, Rueda OM, et al. Copynumber: efficient algorithms for single- and multi-track copy number segmentation. BMC Genomics. 2012;13:1-16. https://doi.org/10.1186/14 71-2164-13-591.

28. Van Loo P, Nordgard SH, Lingjaerde OC, Russnes HG, Rye IH, Sun W, et al. Allele-specific copy number analysis of tumors. Proc Natl Acad Sci U S A. 2010;107:16910-5. doi:1009843107 [pii]. https://doi.org/10.1073/pnas.1 009843107.

29. Pladsen AV, Nilsen G, Rueda OM, Aure MR, Borgan $\varnothing$, Liestøl K, et al. DNA copy number motifs are strong and independent predictors of survival in breast cancer. Commun Biol. 2020;3:1-9.

30. Core R. Team. R : a language and environment for statistical. Computing. 2014;1. https://doi.org/10.1007/978-3-540-74686-7.

31. Han X, Tan Q, Yang S, Li J, Xu J, Hao X, et al. Comprehensive Profiling of Gene Copy Number Alterations Predicts Patient Prognosis in Resected Stages I-III Lung Adenocarcinoma. Front Oncol. 2019;9:1-10.

32. Broët $P$, Camilleri-Broët $S$, Zhang $S$, Alifano $M$, Bangarusamy $D$, Battistella $M$, et al. Prediction of clinical outcome in multiple lung cancer cohorts by integrative genomics: implications for chemotherapy selection. Cancer Res. 2009;69:1055-62.

33. Eischen CM, Lozano G. The Mdm network and its regulation of p53 activities: a rheostat of cancer risk. Hum Mutat. 2014;35:728-37. https://doi. org/10.1002/humu.22524.
34. Berger AH, Imielinski M, Duke F, Wala J, Kaplan N, Shi G-X, et al. Oncogenic RIT1 mutations in lung adenocarcinoma. Oncogene. 2014;33(35):4418-23 November 2013:1-6. https://doi.org/10.1038/onc.2013.581.

35. Diaz-Garcia CV. Agudo-Lopez a., Perez C, Lopez-Martin J a., RodriguezPeralto JL, de Castro J, et al. DICER1, DROSHA and miRNAs in patients with non-small cell lung cancer: implications for outcomes and histologic classification. Carcinogenesis. 2013;34:1031-8. https://doi.org/10.1093/carcin/ bgt022.

36. Czubak K, Lewandowska MA, Klonowska K, Kowalewski J, Figlerowicz M, Kozlowski P. High copy number variation of cancer-related microRNA genes and frequent amplification of DICER1 and DROSHA in lung cancer. Oncotarget. 2015;6:23399-416.

37. Varella-Garcia M. Chromosomal and genomic changes in lung cancer. Cell Adh Migr. 2009;4:100-6. https://doi.org/10.4161/cam.4.1.10884.

38. Taylor AM, Shih J, Ha G, Gao GF, Zhang X, Berger AC, et al. Genomic and Functional Approaches to Understanding Cancer Aneuploidy. Cancer Cell. 2018;33:676-689.e3.

39. AL Zeyadi M, Dimova I, Ranchich V, Rukova B, Nesheva D, Hamude Z, et al. Whole genome microarray analysis in non-small cell lung cancer. Biotechnol Biotechnol Equip 2015;29:111-118. doi:https://doi.org/10.1080/13102818.2 014.989179.

40. Silwal-Pandit L, Vollan HKM, Chin SF, Rueda OM, McKinney S, Osako T, et al. TP53 mutation spectrum in breast cancer is subtype specific and has distinct prognostic relevance. Clin Cancer Res. 2014;20:3569-80.

41. Knudson AG. Mutation and cancer: statistical study of retinoblastoma. Proc Natl Acad Sci U S A. 1971;68:820-3.

42. Assoun S, Theou-Anton N, Nguenang M, Cazes A, Danel C, Abbar B, et al. Association of TP53 mutations with response and longer survival under immune checkpoint inhibitors in advanced non-small-cell lung cancer. Lung Cancer. 2019;132:65-71. https://doi.org/10.1016/j.lungcan.2019.04.005.

43. Shepherd FA, Pereira JR, Ciuleanu T, Tan EH, Hirsh V, Thongprasert S, et al. Erlotinib in previously treated non-small-cell lung Cancer. N Engl J Med. 2005;353:123-32.

44. Hirsch FR. Varella-Garcia M, Bunn P a, Di Maria M V, Veve R, Bremmes RM, et al. epidermal growth factor receptor in non-small-cell lung carcinomas: correlation between gene copy number and protein expression and impact on prognosis. J Clin Oncol. 2003;21:3798-807.

45. Hirsch FR. Paz- L, Mok TS, Jänne P a, Eberhardt WE, Cappuzzo F, et al. epidermal growth factor receptor inhibition in lung Cancer status 2012. J Thorac Oncol. 2013;8:373-84. https://doi.org/10.1097/JTO.0b013e31827edoff.

46. Yuan S, Yu SL, HYWHWY C, Hsu YC, Su KY, HYWHWY C, et al. Clustered genomic alterations in chromosome $7 p$ dictate outcomes and targeted treatment responses of lung adenocarcinoma with EGFR-activating mutations. J Clin Oncol. 2011;29:3435-42. doi:JCO.2011.35.3979 [pii]. https:// doi.org/10.1200/JCO.2011.35.3979.

47. Planck M, Edlund K, Botling J, Micke P, Isaksson S, Staaf J. Genomic and transcriptional alterations in lung adenocarcinoma in relation to EGFR and KRAS mutation status. PLoS One. 2013;8:1-14.

48. Wang Q, Diskin S, Rappaport E, Attiyeh E, Mosse Y, Shue D, et al. Integrative genomics identifies distinct molecular classes of neuroblastoma and shows that multiple genes are targeted by regional alterations in DNA copy number. Cancer Res. 2006;66:6050-62. https://doi.org/10.1158/0008-5472.CA $\mathrm{N}-05-4618$.

49. Heidenblad M, Lindgren D. Veltman J a, Jonson T, Mahlamäki EH, Gorunova L, et al. microarray analyses reveal strong influence of DNA copy number alterations on the transcriptional patterns in pancreatic cancer: implications for the interpretation of genomic amplifications. Oncogene. 2005;24:1794-801.

50. Sholl LM, Aisner DL, Varella-Garcia M, Berry LD, Dias-Santagata D, Wistuba II, et al. Multi-institutional oncogenic driver mutation analysis in lung adenocarcinoma. J Thorac Oncol. 2015;10:768-77. https://doi.org/10.1097/ JTO.0000000000000516.

51. Collisson E A., Campbell JD, brooks AN, Berger AH, lee W, Chmielecki J, et al. comprehensive molecular profiling of lung adenocarcinoma. Nature. 2014; 511:543-550. doi:https://doi.org/10.1038/nature13385, 7511.

52. Hudes G, Carducci M, Tomczak P, Dutcher J, Figlin R, Kapoor A, et al. Temsirolimus, interferon alpha or both for advanced renal cell carcinoma. N Engl J Med. 2007;356:2271-81.

53. Vansteenkiste J, Canon J-L, De Braud F, Grossi F, De Pas T, Gray J, et al. Safety and efficacy of Buparlisib (BKM120) in patients with untreated squamous non-small cell lung Cancer. J Thorac Oncol. 2015;10:1319-27. https://doi.org/10.1097/JTO.0000000000000607. 
54. Sun Z, Wang Z, Liu X, Wang D. New development of inhibitors targeting the PI3K/AKT/mTOR pathway in personalized treatment of non-small-cell lung cancer. Anti-Cancer Drugs. 2015;1:1-14. https://doi.org/10.1097/CAD. 0000000000000172.

55. Ekman S, Wynes MW, Hirsch FR. The mTOR pathway in lung Cancer and implications for therapy and biomarker analysis. J Thorac Oncol. 2012;7:947-53.

56. Jensen TJ, Goodman AM, Kato S, Ellison CK, Daniels GA, Kim L, et al. Genome-wide sequencing of cell-free DNA identifies copy-number alterations that can be used for monitoring response to immunotherapy in Cancer patients. Mol Cancer Ther. 2018;18:448-58.

\section{Publisher's Note}

Springer Nature remains neutral with regard to jurisdictional claims in published maps and institutional affiliations.

- fast, convenient online submission

- thorough peer review by experienced researchers in your field

- rapid publication on acceptance

- support for research data, including large and complex data types

- gold Open Access which fosters wider collaboration and increased citations

- maximum visibility for your research: over $100 \mathrm{M}$ website views per year

At BMC, research is always in progress.

Learn more biomedcentral.com/submissions 